\title{
Historique du dépistage des conduites addictives en milieu professionnel en Europe et aux États-Unis
}

\section{History of workplace drug testing : European and American aspects}

(1) Hôpital Universitaire, Laboratoire de Biologie Clinique, De Pintelaan 185 - B-9000 GENT, Belgique

(2) Institut de Médecine Légale, place Théo Varlet - F-59000 LILLE, France

* Auteur à qui adresser la correspondance : Alain VERSTRAETE, Laboratoire de Biologie Clinique,

De Pintelaan 185 - B-9000 GENT, Belgique - Tél : +32 92403407 - Fax : +32 92404985

e-mail : alain.verstraete@ rug.ac.be

(Reçu le $1^{\text {er }}$ février 2002 ; accepté le 15 février 2002)

\section{RÉSUMÉ}

L'histoire des dépistages de drogues en milieu professionnel commence au début des années 80 aux États-Unis. Différents éléments (un accident sur le porte-avions Nimitz où 9 des 14 matelots décédés étaient positifs pour les métabolites du cannabis, différents accidents de chemin de fer où la drogue était impliquée et l'épidémie de crack très médiatisée) ont contribué à la sensibilisation au problème de l'usage de la drogue dans le milieu professionnel. En 1986, le président Reagan a promulgué un Executive Order qui stipulait que tous les employés fédéraux ayant une fonction avec un aspect de sécurité devraient subir régulièrement un dépistage de drogues. En 1988 sont parus des guidelines pour ces dépistages : certification des laboratoires, chaîne de qualité, contrôle de qualité, dépistage par immunoessai et confirmation par chromatographie en phase gazeuse couplée à la

\section{SUMMARY}

The history of workplace drug testing starts at the beginning of the Eighties in the United States. Various elements (an accident on the Nimitz aircraft carrier in which 9 of the 14 killed sailors were positive for cannabis metabolites, various railway accidents where drugs were involved and the very mediatised crack epidemic) contributed to the development of the use of workplace drug testing. In 1986, president Reagan signed an Executive Order which stipulated that all federal employees in safety sensitive positions should undergo regular drug testing.

In 1988 guidelines for these tests appeared : certification of the laboratories, chain of custody, quality control, screening by immunoassay and confirmation by gas chromatographymass spectrometry, In 1990, the American department of transport subjected the workers of the road industry, the air- 
spectrométrie de masse. En 1990, le ministère des transports américain soumettait les travailleurs de l'industrie du transport routier, aérien, maritime et de l'industrie des pipelines aux mêmes contrôles. Les guidelines ont été adaptés au début de 2000, ils sont parus au format FAQ (frequently asked questions), et les dépistages pour contrôler la validité de l'échantillon deviennent obligatoires. En 2001, 30 à 40 millions de travailleurs américains ont été contrôlés, pour un coût total de 1,5 milliards de dollars. Ces dernières années, le nombre d'entreprises qui contrôlent les travailleurs a légèrement diminué, probablement à cause de la difficulté à trouver des employés ou ouvriers en nombre suffisant. Certains rapports assez critiques sur les aspects coût/bénéfice ont été publiés. De plus les laboratoires sont confrontés au problème de l'adultération qui nécessite beaucoup de dépistages supplémentaires. Ces facteurs ont contribué à la préparation de nouveaux guidelines qui prévoient de pouvoir tester d'autres échantillons (cheveux, salive et sueur) et donnent la possibilité d'utiliser des tests de dépistage rapides sous conditions très strictes.

En Europe, il y a très peu d'information disponible, mais les dépistages de drogues sur le lieu de travail sont beaucoup plus rares. Ils sont les plus répandus au Royaume-Uni et dans les pays scandinaves. Il n'y a pas de législation spécifique. Beaucoup d'entreprises établissent une politique vis à vis des drogues sans prévoir les tests, et souvent les drogues sont dépistées par des tests rapides, sans confirmation des positifs ni contrôle de qualité. Des guidelines au RoyaumeUni ont servi de base à ceux de l'European Workplace Drug Testing Society (EWDTS), société savante ayant pour objet d'assurer que le contrôle des drogues en milieu professionnel en Europe est accompli selon un standard de qualité défini, d'une façon qui peut être défendue en justice, et de fournir un forum indépendant pour tous les aspects du contrôle des drogues sur les lieux de travail. En Europe, le pourcentage de positifs est variable, mais semble diminuer dans les années qui suivent l'introduction des dépistages. Le cannabis est la drogue la plus souvent retrouvée.

De nombreux efforts seront donc nécessaires en Europe pour faire accepter les dépistages de drogues en milieu professionnel, pour établir et maintenir la confiance dans les résultats de laboratoire et pour donner un statut légal à ces dépistages tout en préservant la vie privée et les droits des employés.

\section{MOTS-CLÉS}

Drogue, dépistage, milieu professionnel, historique.

\section{Introduction}

Les tests de dépistage de drogues en milieu professionnel ont été utilisés dans l'armée américaine dès la guerre du Viêt-Nam. Au cours des années, ils ont également été utilisés dans les services fédéraux, dans les entreprises de transport et dans la majorité des grandes entreprises américaines. On considère que dans ce domaine, l'Europe a un retard de 10 à 15 ans, mais les tests de dépistage de drogues en milieu professionnel y sont de plus en plus utilisés. Dans cet article, nous ferons l'historique de l'utilisation de ces tests aux États-Unis et en Europe. lines, maritime transport industry and pipelines to the same controls. The guidelines were adapted at the beginning of 2000, they appeared in FAQ (frequently asked questions) format, and specimen validity tests were also required. In 2001, 30 to 40 million American workers were controlled, at a total cost of 1.5 billion dollars. These last years, the number of companies which control the workers slightly fell, probably because of the difficulty in finding a sufficient number of employees. Certain rather critical reports appeared, especially on the cost benefit aspect. Moreover laboratories are dealing with the problem of the adulteration which requires many additional tests. These factors contributed to the preparation of new guidelines that include testing other samples (hair, saliva and sweat patches) and give the possibility of using (under very strict conditions) onsite screening tests.

In Europe, there is very little information available, but workplace drug tests are much rarer. They are most widespread in the United Kingdom and in the Scandinavian countries. There is no specific legislation. Many companies establish a drugs policy without drug testing, and often drugs are detected by onsite tests, without confirmation of positives nor the quality control. In the United Kingdom, guidelines have been adopted, which have served as the base of the guidelines of the European Workplace drug testing society or EWDTS which is a scientific society founded with the aim of ensuring that workplace drug testing in Europe is accomplished according to a definite standard of quality and in a legally defensible way, and to provide an independent forum for all the aspects of workplace drug testing. In Europe, the percentage of positives is variable, but seems to decrease in the years which follow the introduction of the tests. Cannabis is most often found drug.

Many efforts will be needed to establish workplace drug testing as an accepted part of a company policy on drugs: establishing and maintaining the confidence in the results of the laboratory, establishing the legal status of WDT, preserving the privacy and rights of the employees.

\section{KEY-WORDS}

Drug testing, workplace, guidelines, history.

\section{Les années soixante et soixante-dix}

Aux États-Unis, les premiers dépistages de drogues à grande échelle ont été faits par les militaires. Pendant le guerre du Viêt-Nam, l'armée américaine a été confrontée au problème de l'abus de drogues par les soldats. $\mathrm{Au}$ départ l'armée avait introduit le dépistage des drogues pour identifier les utilisateurs d'hérö̈ne qui rentraient aux États-Unis après leur service militaire au Viêt-Nam. Ce programme a été étendu aux soldats avant leur incorporation au service militaire. A cette 
époque, la méthode la plus courante était le chromatographie en couche mince.

Les premiers efforts technologiques pour le dépistage par immunoessai en phase homogène ont été faits par le firme SYVA (une joint venture de Syntex et de Varian), d'abord en utilisant la méthodologie "Free Radical Assay Technique" (FRAT), très vite remplacée par 1'Enzyme Multiplied Immunoassay Technique (EMIT) à la fin des années 70. Des radioimmunoessais sont aussi apparus rapidement. En effectuant ces analyses sur des automates de laboratoire, les dépistages ont pu être faits rapidement et à moindre coût. Ainsi le ministère de la défense a pu rapidement étendre son programme de dépistage.

\section{Les années quatre-vingt}

\section{Aux États-Unis}

En 1980, le ministère de la défense des Etats-Unis établit que $26 \%$ des militaires utilisaient de la drogue. Parmi les jeunes militaires de 18 à 25 ans, ce pourcentage allait jusqu'à $47 \%$ dans la Navy et chez les Marines. En 1981, un accident d'avion sur le porteavions Nimitz causa la mort de 14 marins. Chez 9 d'entre eux, les analyses toxicologiques démontraient la présence de cannabinoïdes dans le sang. De plus, le pilote de l'avion prenait un antihistaminique sans que son supérieur, ni le médecin du bord ne le sachent. La publicité autour de cet accident a accéléré la décision de la marine de commencer des tests généralisés.

En 1983 les industries du transport commencèrent à s'intéresser au problème, suivies en 1984 par l'industrie pétrolière et l'industrie chimique. En 1986, le gouvernement fédéral entreprit des efforts à grande échelle pour promouvoir le dépistage des drogues sur le lieu du travail. Au printemps 1986, la commission sur le crime organisé publia son rapport sur les attitudes des Américains vis à vis de l'abus et du trafic de drogues et du crime organisé. Après avoir montré la relation entre ces deux phénomènes, la commission a proposé une série de mesures pour tenter de diminuer la demande de drogues. En particulier, elle demandait au gouvernement de "fournir un exemple de l'inadmissibilité de l'usage des drogues". La commission suggéra au Président de demander aux responsables de toutes les agences fédérales de formuler immédiatement des règles d'action claires avec des règles d'implémentation, y compris le dépistage des drogues, pour exprimer l'inadmissibilité totale de l'usage de drogues par les employés fédéraux. Le 15 septembre 1986, le président Reagan a promulgua l'ordre exécutif 12564 sur le Drug Free Federal Workplace. Il déclarait que la drogue cau- sait des milliards de dollars de perte en productivité chaque année et en particulier que les employés fédéraux qui utilisent les drogues illégales étaient moins productifs, moins fiables, et plus enclin à absentéisme. De plus, il donnait mandat à chaque service de l'exécutif d'établir un programme pour "tester l'usage de drogues illégales par les employés fédéraux ayant une fonction comportant un aspect de sécurité". Les agences fédérales ont rapidement établi des programmes de dépistage de drogues. En janvier 1987 survint un accident de train à Chase (Maryland), où 16 passagers furent tués et 174 blessés et qui causa des millions de dollars de dégâts matériels. L'urine du chauffeur et du chef de train étaient positives pour le cannabis. Le 21 janvier 1987, le ministère des transports proposa un programme de dépistage de drogues rigoureux, incluant le dépistage des drogues à l'embauche, après un accident et au hasard chez les pilotes de ligne, travailleurs des chemins de fer, contrôleurs aériens et autres employés dans des postes de sécurité (1).

Les dépistages de drogues ont été attaqués en justice par différentes organisations. Entre 1987 et 1991, le ministère de la justice américain a dépensé 725000 dollars pour défendre la législation sur les tests de drogues. Deux cas ont été jusqu'à la Cour Suprême, où une décision a été prise le 21 mars 1989. Dans les deux cas, la Cour Suprême a soutenu la légalité du programme de dépistage de drogues du gouvernement, opposant les droits au respect de la vie privée des d'employés contre "d'autres intérêts irrésistibles du gouvernement".

En 1998, le ministère de la Santé a établi des guidelines : les analyses ne peuvent être effectuées que par des laboratoires certifiés, une chaîne de qualité doit être garantie, et il doit y avoir des normes très strictes pour le contrôle de qualité (2). Au départ, les guidelines stipulaient de dépister au moins le cannabis et la cocaïne, de faire un dépistage par immunoessai, suivi d'une confirmation des positifs par chromatographie en phase gazeuse couplée à la spectrométrie de masse (CG/SM). En plus du secteur public et des transports, beaucoup d'entreprises commençaient à contrôler leur personnel, en partie pour pouvoir obtenir des contrats avec le gouvernement, mais aussi pour des raisons de responsabilité civile ou financières. Différentes études de rentabilité de ces programmes ont été effectuées, avec des résultats parfois contradictoires. Une des études les plus citées est celle des services postaux, qui entre septembre 1987 et mai 1988 ont fait des tests de dépistage à l'embauche chez plus de 5000 personnes, mais ont néanmoins embauché les $9 \%$ qui étaient positifs. Les trois années suivantes, différents paramètres comme l'absentéisme, la rotation du personnel, les renvois au 
service d'assistance aux employés (Employee Assistance Program, EAP) les coûts médicaux et les mesures disciplinaires, ont été suivis. L'absentéisme était de $11,4 \%$ chez les employés dont le test de dépistage de drogues avait été positif, et $6,85 \%$ chez les négatifs. La rotation du personnel était $77 \%$ plus élevée chez les positifs. Quatorze pour cent des positifs ont été renvoyés à l'EAP, contre 2,7\% des négatifs. Les frais médicaux étaient supérieurs de $83 \%$ chez les positifs et les sanctions disciplinaires 2,4 fois plus nombreuses. Les auteurs de l'étude ont estimé qu'en dix ans 100 millions de dollars auraient pu être épargnés si on n'avait pas engagé les positifs, soit environ 19000 dollars par employé (3).

\section{En Europe}

En 1989, pendant la présidence espagnole de l'Union Européenne, un texte a été adopté concernant la fiabilité des dépistages de drogues. Certaines entreprises, surtout d'origine américaine, ont commencé à tester leurs employés, par exemple dans l'industrie du pétrole.

\section{Les années quatre-vingt-dix Aux États-Unis}

En 1990, le ministère des Transports a établi des règles pour tester les travailleurs dans l'industrie du transport routier, aérien, maritime et des oléoducs. En 1994, ces règles ont été étendus aux transports en commun.

Les guidelines pour les employés fédéraux ont été modifiés deux fois : une fois pour abaisser le seuil pour les cannabinoïdes de 100 à $50 \mathrm{ng} / \mathrm{mL}$ et une fois pour augmenter le seuil pour la morphine de 300 à 2000 $\mathrm{ng} / \mathrm{mL}$ (de plus, la confirmation n'était considérée comme positive que si la 6-monoacétylmorphine était présente à plus de $10 \mathrm{ng} / \mathrm{mL}$ ). Cette modification a été apportée pour diminuer le nombre de faux positifs. On avait en effet observé que $87 \%$ des sujets trouvés opiacés positifs étaient considérés comme négatifs par les Medical Review Officers (MRO), parce que cela pouvait correspondre à une prise de médicaments ou de pâtisserie contenant des graines de pavot. Ces nouveaux seuils ont eu pour conséquence une diminution de moitié du nombre de positifs aux opiacés (4).

Dans les années 90, on a vu une augmentation des critiques vis-à-vis des programmes de contrôles de drogues en milieu professionnel. En 1994, il y eut un rapport critique de la National Academy of Sciences (5) et en 1999 l'American Civil Liberties Union (ACLU) publia un rapport intitulé "Drugtesting, a bad investment" (9). Le nombre d'entreprises qui teste les employés atteignit son maximum en 1996 (81\%), pour diminuer plus tard $(67 \%$ en 2001). Le nombre d'échantillons positifs suivit une tendance inverse, passant de $13,6 \%$ en 1988 à 4,6\% en 1999. On observe une petite augmentation en $2000(4,7 \%)$. Le taux de chômage très bas aux Etats-Unis fait qu'il devient difficile de trouver du personnel, expliquant ainsi que certaines firmes ne font plus de dépistage des drogues à l'embauche pour ne pas décourager les candidats. Une étude des services postaux montre qu'un tiers des personnes embauchées a trouvé un nouvel employeur dans les 24 heures nécessaires pour attendre le résultat du test de dépistage de drogues. Certaines entreprises utilisent donc des tests rapides : si le résultat est négatif, l'employé est engagé immédiatement et peut signer son contrat tout de suite.

Un nouveau problème apparaît : la substitution et l'adultération des échantillon, en vue d'obtenir des résultats négatifs. Tout un commerce (entre autres sur Internet) s'organise pour vendre les adultérants. De leur côté, les laboratoires et le législateur réagissent : ils développent de nouveaux tests de dépistage des adultérants et certains tests supplémentaires de dépistage de l'adultération ou de la dilution sont rendus obligatoires. Dans certain cas, on recherche plus les adultérants que les drogues, ce qui augmente sensiblement les coûts.

On a aussi observé un changement dans la philosophie des programmes dans les entreprises : punitive dans les années 80 , elle devient plus positive et curative dans les années 90 . Il faut aider l'employé à arrêter l'usage de drogues ce qui le rendra plus productif pour l'entreprise ("licencier l'usage de drogues, mais garder l'employé").

\section{En Europe}

Il n'y a pas beaucoup de données sur le dépistage des drogues en entreprise en Europe et il est difficile d'obtenir des informations fiables. Les entreprises ne donnent pas volontiers de détails sur leur politique en matière de drogue et seules des statistiques minimales sont disponibles. Il n'y a aucune législation spécifique sur ces tests de dépistage de drogues en milieu professionnel et aucun organisme co-ordinateur officiel n'existe. Souvent le dépistage est réalisé sur place avec des tests rapides, sans confirmation systématique des positifs. Les dépistages en milieu professionnel semblent être les plus développés au Royaume-Uni (on estime que $10 \%$ des grandes entreprises testent leurs employés) et en Scandinavie. Une enquête de marché en 1996 a estimé le potentiel européen de tests de dépistage de drogues à 4 millions par an.

En 1993, une enquête de l'Union Européenne et du 
Bureau International du Travail montre que les dépistages de drogues en milieu professionnel sont peu pratiquées. Plus de $80 \%$ des entreprises ne testent pas à l'embauche. Seuls $27 \%$ des entreprises peuvent effectuer des tests, souvent seulement après un accident, ou comme part d'un programme de traitement. Vingt-huit des 237 organisations consultées ont envisagé d'introduire les tests de dépistage, mais ne l'ont pas fait pour des considérations légales ou apparentées aux droits de l'homme ou parce qu'ils craignaient un effet néfaste sur les relations entre employés et employeurs (7).

En 1993 et 1994, deux exercices de contrôle de qualité ont eu lieu. Ils ont démontré la nécessité de standardisation et de critères d'évaluation (seuils de positivité). Seuls $10 \%$ des laboratoires européens sont capables de faire le dépistage, la confirmation et la quantification de drogues.

En 1996, un groupe d'experts s'est réuni à Barcelone pour établir des recommandations (8), contenant entre autres des propositions de seuils pour le dépistage et pour la confirmation (tableaux I et II).
En 1998 eut lieu le premier congrès "Drugtesting at workplace" en Europe, qui réunissait des participants de 22 pays. Un rapport de ce congrès a été publié (9). Parmi les conclusions, on note que l'Europe à un retard de 10 à 15 ans sur les États-Unis. On y demande une accréditation obligatoire pour les laboratoires et une évaluation de l'impact des programmes de dépistage de drogues sur le nombre d'accidents, afin de convaincre le public et le monde politique que de tels programmes sont bénéfiques. Suite à cette réunion, un groupe informel de personnes intéressées par les tests de dépistage de drogues en milieu professionnel a été formé. En 1999, ce groupe a crée l'European Workplace Drug Testing Society (EWDTS, www.ewdts.org), qui a pour but d'assurer que le contrôle des drogues sur les lieux de travail en Europe est accompli selon un standard de qualité défini, d'une façon qui peut être défendue en justice et de fournir un forum indépendant pour tous les aspects du contrôle des drogues sur les lieux de travail. L'EWDTS a organisé un second congrès à Rimini (Italie) en octobre 2001, qui a réuni 80 experts de nombreux pays.

Tableau I : Comparaison des seuils de dépistage (en $\mathrm{ng} / \mathrm{mL}$ ) de certaines drogues selon différentes recommandations pour le dépistage des drogues en milieu professionnel.

\begin{tabular}{|l|c|c|c|c|c|}
\hline & $\begin{array}{c}\text { Europe } \\
{[\mathbf{8}]}\end{array}$ & $\begin{array}{c}\text { USA } \\
{[\mathbf{1 3}]}\end{array}$ & $\begin{array}{c}\text { Australie } \\
{[\mathbf{1 4}]}\end{array}$ & $\begin{array}{c}\text { Royaume-Uni } \\
{[\mathbf{1 2}]}\end{array}$ & $\begin{array}{c}\text { Proposition } \\
\text { SAMHSA 2001 } \\
{[\mathbf{4}]}\end{array}$ \\
\hline Amphétamines & 300 & 1000 & 300 & 300 & 500 \\
\hline Cannabinoïdes & 50 & 50 & 50 & 50 & 50 \\
\hline Cocaïne & 300 & 300 & 300 & 300 & 150 \\
\hline Opiacés & 300 & 2000 & 300 & 300 & 2000 \\
\hline Phencyclidine & - & 25 & - & 25 & 25 \\
\hline
\end{tabular}

Tableau II : Comparaison des seuils de confirmation (en $\mathrm{ng} / \mathrm{mL}$ ) dans différentes recommandations concernant le dépistage de drogue en milieu professionnel.

\begin{tabular}{|l|c|c|c|c|c|}
\hline & $\begin{array}{c}\text { Europe } \\
{[\mathbf{8}]}\end{array}$ & $\begin{array}{c}\text { USA } \\
{[\mathbf{1 3}]}\end{array}$ & $\begin{array}{c}\text { Australie } \\
{[\mathbf{1 4}]}\end{array}$ & $\begin{array}{c}\text { Royaume-Uni } \\
{[\mathbf{1 2}]}\end{array}$ & $\begin{array}{c}\text { Proposition } \\
\text { SAMHSA 2001 } \\
{[\mathbf{4}]}\end{array}$ \\
\hline Amphétamine & 200 & 500 & 300 & 200 & 250 \\
\hline Methamphétamine & 200 & 500 & 300 & 200 & 250 \\
\hline MDMA, MDEA, MDA & 200 & - & 300 & 200 & 250 \\
\hline 11-nor-9-COOH- $\Delta^{9}$-THC & 15 & 15 & 15 & 15 & 15 \\
\hline Benzoylecgonine & 150 & 150 & 150 & 150 & 100 \\
\hline Morphine & 200 & 2000 & 300 & 300 & 2000 \\
\hline Codéine & - & 2000 & 300 & 300 & 2000 \\
\hline 6-acétylmorphine & - & 10 & 10 & 10 & 10 \\
\hline Dihydrocodéine & - & - & - & 300 & - \\
\hline
\end{tabular}


Une comparaison des taux de positifs enregistrés dans les compagnies de chemins de fer de différent pays illustre bien l'importance du problème, la situation assez comparable dans tous les pays, et la diminution du nombre de positifs au cours des années suivant l'introduction du dépistage (tableau III)

En l'an 2000, dans le cadre d'une loi sur la vie privée, le parlement finlandais à étudié une proposition de loi régissant le dépistage de drogues en milieu professionnel, mais ce chapitre a été retiré juste avant la discussion. La situation en Europe a été revue récemment (10).

\section{Au niveau international}

Le Bureau International du Travail a publié un recueil de directives pratiques, adopté en 1995, qui contient des conseils pour ceux qui doivent prendre en charge les problèmes liés à l'alcoolisme et à la toxicomanie sur le lieu de travail. Il invite les employeurs et les travailleurs à élaborer ensemble une stratégie, définit l'abus de substances toxiques comme un problème médical qu'il convient de traiter de la même manière que toute autre maladie, recommande la prise en compte de ce problème dans la gestion au sens large et démontre que discipliner les personnes qui abusent de substances toxiques est un impératif moral (11).

Tableau III : Pourcentage de dépistages positifs à l'embauche dans les entreprises de chemins de fer de différents pays.

\begin{tabular}{|l|c|c|}
\hline \multicolumn{1}{|c|}{ Pays } & Année & Pourcentage de positifs \\
\hline Belgique & 2001 & $7,2 \%$ \\
\hline Allemagne & 2000 & $5,8 \%$ \\
\hline Royaume-Uni & $1994-1997$ & $0,18-0,36 \%$ \\
\hline \multirow{3}{*}{ Espagne } & $1988-1992$ & $3,6 \%$ \\
\cline { 2 - 3 } & $1993-1995$ & $1,4 \%$ \\
\cline { 2 - 3 } & $1996-1997$ & $1,1 \%$ \\
\hline \multirow{2}{*}{ Suéde } & 1998 & $0,9 \%$ \\
\cline { 2 - 3 } & 1999 & $0,2 \%$ \\
\hline \multirow{2}{*}{ États-Unis } & 1998 & $0,8 \%$ \\
\hline
\end{tabular}

\section{Le début du vingt-et-uniè- me siècle.}

\section{Aux États-Unis}

En 2000, les guidelines du ministère des Transports ont été modifiées. Les modifications les plus importantes sont le format question-réponse et le fait que les laboratoires doivent effectuer des tests de validité de l'échantillon.
Le dépistage des drogues en milieu professionnel est devenu extrêmement important aux États-Unis. Environ 30 à 40 millions de travailleurs sont contrôlés chaque année. Ces tests sont obligatoires chez les fonctionnaires fédéraux, dans le secteur du transport et dans l'industrie nucléaire. Ces contrôles sont aussi effectués dans la majorité des grandes entreprises. En mai 2001, 61 laboratoires étaient certifiés. L'industrie des réactifs nécessaires au dépistage des drogues a un chiffre d'affaires de 1,5 milliards de dollars (800 millions pour la collecte des échantillons, 725 millions pour les analyses et 38 millions pour les MRO).

Un groupe de travail prépare encore une nouvelle version des guidelines pour les services fédéraux. Les modifications les plus importantes sont la recherche de nouvelles drogues comme l'ecstasy et autres dérivés des amphétamines, l'abaissement des seuils pour les amphétamines et la cocaïne, la possibilité de tester d'autres échantillons comme la salive, la sueur, les cheveux et la possibilité d'utiliser des tests rapides sur le terrain. Les tests de dépistage rapides pourraient être utilisés pour le dépistage dans les urines ou la salive, à condition d'être approuvés par la Food and Drug Administration (FDA) et par la Health and Human Services Administration. Les fabricants de tests doivent être certifiés et leurs installations doivent être inspectées une fois par an. En cas d'utilisation de tests rapides, le contrôle de qualité doit inclure chaque jour un test positif et un test négatif, ou un test positif après chaque échantillon positif. De plus un échantillon négatif sur vingt doit être envoyé à un laboratoire certifié pour y être retesté. Les personnes qui font les tests doivent être formés à l'exécution et à l'interprétation des tests.

\section{En Europe.}

Un groupe de travail britannique a établi les United Kingdom Laboratory guidelines for legally defensible workplace drug testing (12). Ces guidelines ont été proposées à l'EWDTS, pour servir de base aux guidelines européens. Un groupe de travail commun est chargé de les actualiser et de poursuivre le travail sur d'autres sujets, notamment tests sur les matrices alternatives.

\section{Conclusion}

Les tests de dépistage de drogues en milieu professionnel se sont surtout développés aux États-Unis à la fin des années 80. L'Europe à 10 à 15 ans de retard.

Ces tests sont encore controversés, surtout en Europe, où il n'y a pas de législation spécifique. Cependant on 
y observe une augmentation de la fréquence d'utilisation de ces tests, qui va probablement continuer dans les prochaines années. Récemment, pour répondre à certaines critiques, de nouvelles orientations sont apparues, comme l'usage d'autres échantillons que le sang et l'urine et la possibilité d'utiliser des tests de dépistage sur le terrain.

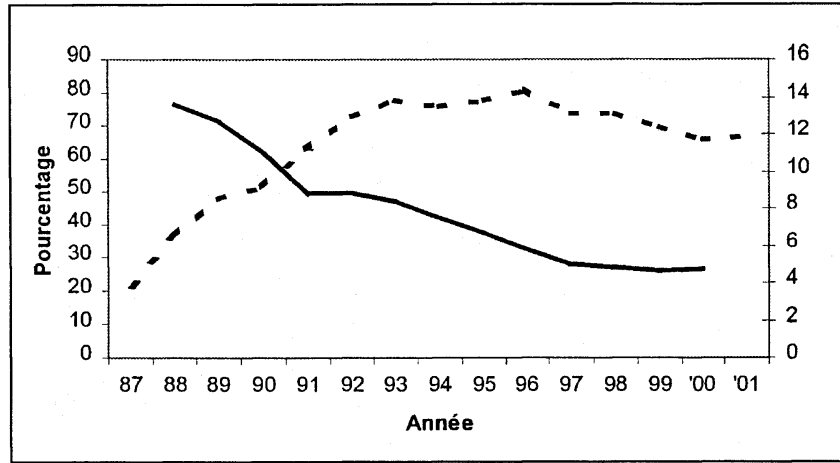

Figure 1 : Proportion de grandes sociétés américaines qui effectuent le dépistage de drogues lors de l'embauche (ligne pointillée, échelle de gauche ; source : American Management Association) et proportion de tests positifs (ligne continue, échelle de droite ; source : Quest Diagnostics). Cette figure montre bien la relation inverse entre le nombre d'entreprises qui font les tests et le nombre de positifs.

\section{Références}

1. Zwerling C. Current practice and experience in drug and alcohol testing in the workplace. Bulletin on Narcotics, 1.1.1993, 155-96 http : //www.undcp.org/bulletin/bulletin_1993-01-01_2_page006.html.

2. Mandatory guidelines for federal workplace drugtesting programs. Fed. Reg. 1988 ; 53 : 11970-89.

3. Peat M.A. Financial viability of screening for drugs of abuse. Clin. Chem. 1995 ; 41 : 805-8.

4. Peat M. Workplace drug testing - The good, the bad and the ugly. In : Rasanen I., ed. Proceedings of TIAFT 2000. Helsinki : TIAFT, 2001 : 130-40.

5. Normand J. Under the influence ? Drugs and the American workforce. Washington, DC : National Academy Press 1994.

6. Maltby L.L. Drug Testing. A bad investment. New York. American Civil Liberties Union, 1999 : 1-28.

7. Smith J. P. Alcohol and drugs in the workplace : attitudes, policies and programmes in the European Community. Genève : Bureau International du Travail 1993 : 1-76.

8. Kintz P., de la Torre R., Segura J., De Zeeuw R., Willliams J. and the Toxicology Experts Working Group. Recommendations for the reliable detection of illicit drugs in urine within the European Union, with special attention to the workplace. Toxicorama $1997 ; 11: 197$ 202.

9. Dalen P., Beck O., Bergman U., Björklöv P., Finer D., Garle M., Sjökvist F. Workplace drug testing (WDT) likely to increase in Europe. Report from the first european symposium on WDT including selected abstracts. Eur. J. Clin. Pharmacol. 2000 ; 56 : 103-20.

10. Verstraete A.G., Pierce A. Workplace drug testing in Europe. Forensic Sci. Int. 2001 ; 121 ; 2-6.

11. Management of alcohol- and drug-related issues in the workplace. Geneva, International Labour Office, 1996, $1-61$.

12. UK Steering Group on Workplace Drug Testing. United Kingdom Laboratory guidelines for legally defensible workplace drug testing. (Peut être téléchargé de : www.londontox.org).

13. Mandatory guidelines for federal workplace drug testing programs. June 9, 1994 revision, Federal Register 59 (1194) 29908.

14. Recommended practice for the collection, detection and quantitation of drugs of abuse. AS 4308 - 1995. Standards Australia, Homebush NSW 2140. 